\title{
SOLITON SOLUTIONS TO RESONANT NONLINEAR SCHRODINGER'S EQUATION WITH TIME-DEPENDENT COEFFICIENTS BY MODIFIED SIMPLE EQUATION METHOD
}

\author{
Ahmed H. Arnous ${ }^{1}$, Mohammad Mirzazadeh ${ }^{2}$, Qin Zhou ${ }^{3}$, \\ Seithuti P. Moshokoa ${ }^{4}$, Anjan Biswas ${ }^{4,5}$ \& Milivoj Belic ${ }^{6}$ \\ ${ }^{1}$ Department of Engineering Mathematics and Physics, \\ Higher Institute of Engineering, El Shorouk, Egypt \\ 2 Department of Engineering Sciences, Faculty of Technology and Engineering, \\ East of Guilan, University of Guilan, P.C. 44891-63157, Rudsar-Vajargah, Iran \\ 3 School of Electronics and Information Engineering, \\ Wuhan Donghu University, Wuhan-430212, PR China \\ 4 Department of Mathematics and Statistics, \\ Tshwane University of Technology, Pretoria-0008, South Africa \\ ${ }^{5}$ Faculty of Science, Department of Mathematics, \\ King Abdulaziz University, Jeddah-21589, Saudi Arabia \\ 6 Science Program, Texas A and M University at Qatar, \\ PO Box 23874, Doha, Qatar
}

\begin{abstract}
This paper studies resonant nonlinear Schrodinger's equation with time-dependent coefficients and four forms of nonlinear media. They are Kerr law, power law, parabolic law and dual-power law. Soliton solutions are recovered by the aid of modified simple equation method.
\end{abstract}

OCIS Codes: 060.2310; 060.4510; 060.5530; 190.3270; 190.4370

1

(C) 2016. This manuscript version is made available under the Elsevier user license http://www.elsevier.com/open-access/userlicense/1.0/ 


\section{INTRODUCTION}

Theory of solitons forms the basic fabric in all optical communication systems. There are several developments and advancements that are observed currently [1-20]. A large variety of results are being relentlessly reported across various journals throughout the globe. There are in fact several models that are studied in this context. One of the interesting models that will be revisited in this paper is the resonant nonlinear Schrödinger's equation (RNLSE). While NLSE is well known to describe the propagation of solitons through optical fibers, RNLSE comes with an additional quantum Hall effect [1-9].

It is needless to mention that RNLSE has been already extensively studied by several authors including a few of the current authors of this paper. In the past, this equation was addressed by simplest equation method, semi-inverse variational principle, Kudryashov's scheme, $G^{\prime} / G$-expansion scheme, trial solutions approach, bifurcation analysis, method of undetermined coefficients as well as several other approaches [1-9, $11,12]$. This paper is therefore a revisitation of the same model. This time, RNLSE will be studied with time-dependent coefficients by a different algorithm. This is the modified simple equation approach. The subsequent section is an introduction to this method that will be followed by its application to RNLSE. There are four types of nonlinearity that are studied in this paper. They are cubic or Kerr law, power law, parabolic law and dual-power law.

\section{MODIFIED SIMPLE EQUATION METHOD}

Suppose we have a nonlinear evolution equation in the form

$$
P\left(u, u_{t}, u_{x}, u_{x x}, u_{t t}, u_{t x}, \ldots\right)=0
$$

where $P$ is a polynomial in $u(x, t)$ and its partial derivatives in which the highest order derivatives and nonlinear terms are involved. In the following, we give the main steps of this method [1,2]:

Step-1: We use the transformation

$$
u(x, t)=u(\xi), \quad \xi=x-c t,
$$

where $c$ is a constant to be determined, to reduce Eq. (1) to the following ODE :

$$
Q\left(u, u^{\prime}, u^{\prime \prime}, \ldots\right)=0,
$$

where $Q$ is a polynomial in $u(\xi)$ and its total derivatives, while ${ }^{\prime}=\frac{d}{d \xi}$.

Step-2: We suppose that Eq. (3) has the formal solution.

$$
u(\xi)=\sum_{l=0}^{N} a_{l}\left(\frac{\psi^{\prime}(\xi)}{\psi(\xi)}\right)^{l},
$$

where $a_{l}$ are constants to be determined, such that $a_{N} \neq 0$, and $\psi(\xi)$ is an unknown function to be determined later.

Step-3: We determine the positive integer $N$ in Eq. (4) by considering the homogeneous balance between the highest order derivatives and the nonlinear terms in Eq. (3). 
Step-4: We substitute (4) into (3), then we calculate all the necessary derivatives $u^{\prime}, u^{\prime \prime}, \ldots$ of the unknown function $u(\xi)$ and we account the function $\psi(\xi)$. As a result of this substitution, we get a polynomial of $\frac{\psi^{\prime}(\xi)}{\psi(\xi)}$ and its derivatives. In this polynomial, we gather all the terms of the same power of $\psi^{-j}(\xi), j=0,1,2, \ldots$ and its derivatives, and we equate with zero all the coefficients of this polynomial. This operation yields a system of equations which can be solved to find $a_{k}$ and $\psi(\xi)$. Consequently, we can get the exact solutions of Eq. (1) .

\section{APPLICATION TO RNLSE}

The RNLSE with time-dependent coefficients, which will be studied in this section, is [1-9]

$$
i \psi_{t}+\alpha(t) \psi_{x x}+\beta(t) G\left(|\psi|^{2}\right) \psi+\gamma(t)\left(\frac{|\psi|_{x x}}{|\psi|}\right) \psi=0
$$

Here, in (5), the first term is the linear evolution, while $\alpha(t)$ is the coefficient of group velocity dispersion (GVD) and $\beta(t)$ is the coefficient of nonlinearity. Finally, $\gamma(t)$ is quantum or Bohm potential that appears in the context of chiral solitons in quantum Hall effect. It is also seen in the context of Madelung fluid in quantum mechanics. Also, the functional $G$ meets the following technical condition:

$G$ is a real-valued algebraic function, and it is necessary to have the smoothness of the complex function $G\left(|q|^{2}\right) q: C \rightarrow C$. Considering the complex plane $C$ as a two dimensional linear space $R^{2}$, the function $G\left(|q|^{2}\right) q$ is $k$ times continuously differentiable, so that

$$
G\left(|q|^{2}\right) q \in \bigcup_{m, n=1}^{\infty} C^{k}\left((-n, n) \times(-m, m) ; R^{2}\right) .
$$

The starting point for the analysis of this equation is the traveling wave hypothesis

$$
\psi(x, t)=U(\xi) e^{i(-\kappa x+\omega(t) t)}, \quad \xi=x+2 \kappa \int_{0}^{t} \alpha(t) d t,
$$

so that Eq. (5) transforms to

$$
(\alpha(t)+\gamma(t)) U^{\prime \prime}-\left\{t \frac{d \omega(t)}{d t}+\omega(t)+\kappa^{2} \alpha(t)\right\} U+\beta(t) F\left(U^{2}\right) U=0 .
$$

Eq. (8) will now be analyzed in the next four subsections that will be based on the type of nonlinearity.

\subsection{CUBIC (KERR) NONLINEARITY}

The cubic nonlinearity is the case when $G(s)=s$. This form of nonlinearity typically arises in the context of deep water waves or nonlinear fiber optics when the refractive index of the light is proportional to the intensity. For cubic nonlinearity, RNLS equation with time-dependent coefficients is [1-9]

$$
i \psi_{t}+\alpha(t) \psi_{x x}+\beta(t)|\psi|^{2} \psi+\gamma(t)\left(\frac{|\psi|_{x x}}{|\psi|}\right) \psi=0
$$

In this case, Eq. (9) reduces to

$$
(\alpha(t)+\gamma(t)) U^{\prime \prime}-\left\{t \frac{d \omega(t)}{d t}+\omega(t)+\kappa^{2} \alpha(t)\right\} U+\beta(t) U^{3}=0 .
$$


For the solutions of Eq. (9), assume

$$
U(\xi)=\sum_{l=0}^{N} a_{l}\left(\frac{\psi^{\prime}(\xi)}{\psi(\xi)}\right)^{l}, a_{N} \neq 0,
$$

where $a_{l}$ are all constants to be determined. Balancing $U^{\prime \prime}$ with $U^{3}$ in Eq. (10) gives $N=1$. Consequently, we reach

$$
U(\xi)=a_{0}+a_{1}\left(\frac{\psi^{\prime}(\xi)}{\psi(\xi)}\right)
$$

Substituting Eq. (12) in Eq. (10) and then setting the coefficients of $\psi^{-j}, j=0,1,2,3$ to zero, then we obtain a set of algebraic equations involving $a_{0}, a_{1}, \kappa$ and $\omega(t)$ as following $\psi^{-3}$ Coeff.:

$$
a_{1}\left(2(\alpha(t)+\gamma(t))+\beta(t) a_{1}^{2}\right)\left(\psi^{\prime}\right)^{3}=0,
$$

$\psi^{-2}$ Coeff.:

$$
3 a_{1} \psi^{\prime}\left(\beta(t) a_{0} a_{1} \psi^{\prime}-(\alpha(t)+\gamma(t)) \psi^{\prime \prime}\right)=0
$$

$\psi^{-1}$ Coeff.:

$$
a_{1}\left(-\left(t \frac{d \omega(t)}{d t}+\omega(t)+\alpha(t) \kappa^{2}-3 \beta(t) a_{0}^{2}\right) \psi^{\prime}+(\alpha(t)+\gamma(t)) \psi^{\prime \prime \prime}\right)=0
$$

$\psi^{0}$ Coeff.:

$$
a_{0}\left(-t \frac{d \omega(t)}{d t}-\omega(t)-\alpha(t) \kappa^{2}+\beta(t) a_{0}^{2}\right)=0 .
$$

Solving this system, we obtain

$$
a_{1}= \pm \sqrt{\frac{-2(\alpha(t)+\gamma(t))}{\beta(t)}}, \omega(t)=\frac{1}{t} \int_{0}^{t}\left\{-\alpha(t) \kappa^{2}+\beta(t) a_{0}^{2}\right\} d t,
$$

where $a_{0}$ is a real arbitrary constants. Substituting (17) into Eqs. (14) and (15), we obtain

$$
\begin{gathered}
\psi^{\prime \prime}= \pm \sqrt{\frac{-2 \beta(t)}{\alpha(t)+\gamma(t)}} a_{0} \psi^{\prime}, \\
\psi^{\prime \prime \prime}=-\frac{2 \beta(t) a_{0}^{2}}{\alpha(t)+\gamma(t)} \psi^{\prime} .
\end{gathered}
$$

From Eqs. (18) and (19), we can deduce that

$$
\begin{gathered}
\psi^{\prime}= \pm \frac{1}{a_{0}} \sqrt{\frac{-(\alpha(t)+\gamma(t))}{2 \beta(t)}} c_{1} e^{ \pm \sqrt{\frac{-2 \beta(t)}{\alpha(t)+\gamma(t)}}} a_{0} \xi \\
\psi=\frac{-(\alpha(t)+\gamma(t))}{2 \beta(t) a_{0}^{2}} c_{1} e^{ \pm \sqrt{\frac{-2 \beta(t)}{\alpha(t)+\gamma(t)}} a_{0} \xi}+c_{2} .
\end{gathered}
$$

where $c_{1}$ and $c_{2}$ are constants of integration. Substituting Eqs. (20) and (21) into Eq. (12), we obtain the following new exact solution to Eq. (9)

$$
\begin{aligned}
& \psi(x, t)=\left\{a_{0} \pm \sqrt{\frac{-2(\alpha(t)+\gamma(t))}{\beta(t)}}\left(\frac{ \pm \frac{1}{a_{0}} \sqrt{\frac{-(\alpha(t)+\gamma(t))}{2 \beta(t)}} c_{1} e^{ \pm \sqrt{\frac{-2 \beta(t)}{\alpha(t)+\gamma(t)}} a_{0} \xi}}{\frac{-(\alpha(t)+\gamma(t))}{2 \beta(t) a_{0}^{2}} c_{1} e^{ \pm \sqrt{\frac{-2 \beta(t)}{\alpha(t)+\gamma(t)}} a_{0} \xi}+c_{2}}\right)\right\} \\
& \times e^{i\left(-\kappa x-\int_{0}^{t}\left\{\alpha(t) \kappa^{2}-\beta(t) a_{0}^{2}\right\} d t\right)}
\end{aligned}
$$


If we set $c_{1}=\frac{-2 \beta(t) a_{0}^{2}}{\alpha(t)+\gamma(t)} e^{\sqrt{\frac{-2 \beta(t)}{\alpha(t)+\gamma(t)}} a_{0} \xi_{0}}, c_{2}= \pm 1$, we obtain

$$
\begin{aligned}
& \psi(x, t)= \pm a_{0} \tanh \left[\frac{1}{2} \sqrt{\frac{-2 \beta(t)}{\alpha(t)+\gamma(t)}} a_{0}\left(x+2 \kappa \int_{0}^{t} \alpha(t) d t+\xi_{0}\right)\right] \\
& \times e^{i\left(-\kappa x-\int_{0}^{t}\left\{\alpha(t) \kappa^{2}-\beta(t) a_{0}^{2}\right\} d t\right)} \\
& \psi(x, t)= \pm a_{0} \operatorname{coth}\left[\frac{1}{2} \sqrt{\frac{-2 \beta(t)}{\alpha(t)+\gamma(t)}} a_{0}\left(x+2 \kappa \int_{0}^{t} \alpha(t) d t+\xi_{0}\right)\right] \\
& \times e^{i\left(-\kappa x-\int_{0}^{t}\left\{\alpha(t) \kappa^{2}-\beta(t) a_{0}^{2}\right\} d t\right)} .
\end{aligned}
$$

These solutions are valid for $\beta(t)(\alpha(t)+\gamma(t))<0$.

$$
\begin{aligned}
& \psi(x, t)= \pm a_{0} \tan \left[\frac{1}{2} \sqrt{\frac{2 \beta(t)}{\alpha(t)+\gamma(t)}} a_{0}\left(x+2 \kappa \int_{0}^{t} \alpha(t) d t+\xi_{0}\right)\right] \\
& \times e^{i\left(-\kappa x-\int_{0}^{t}\left\{\alpha(t) \kappa^{2}-\beta(t) a_{0}^{2}\right\} d t\right)}, \\
& \psi(x, t)=\mp a_{0} \cot \left[\frac{1}{2} \sqrt{\frac{2 \beta(t)}{\alpha(t)+\gamma(t)}} a_{0}\left(x+2 \kappa \int_{0}^{t} \alpha(t) d t+\xi_{0}\right)\right] \\
& \times e^{i\left(-\kappa x-\int_{0}^{t}\left\{\alpha(t) \kappa^{2}-\beta(t) a_{0}^{2}\right\} d t\right)} .
\end{aligned}
$$

These solutions are valid for $\beta(t)(\alpha(t)+\gamma(t))>0$.

\subsection{POWER LAW NONLINEARITY}

The power law nonlinearity arises when $G(s)=s^{n}$, where the parameter $n$ is referred to as the nonlinearity parameter. This kind of law appears in the context of plasma physics, trubulence theory and occasionally in the case of nonlinear fiber optics. It needs to be however noted that one must have $0<n<2$ in order to arrest self-focusing singularity and soliton collapse. For power law nonlinearity, the RNLSE is [1-9]

$$
i \psi_{t}+\alpha(t) \psi_{x x}+\beta(t)|\psi|^{2 n} \psi+\gamma(t)\left(\frac{|\psi|_{x x}}{|\psi|}\right) \psi=0 .
$$

In this case, Eq. (27) reduces to

$$
(\alpha(t)+\gamma(t)) U^{\prime \prime}-\left\{t \frac{d \omega(t)}{d t}+\omega(t)+\kappa^{2} \alpha(t)\right\} U+\beta(t) U^{2 n+1}=0 .
$$

To obtain an analytic solution, we use the transformation $U=V^{\frac{1}{2 n}}$ in Eq. (28) to find

$$
\begin{aligned}
& (\alpha(t)+\gamma(t))\left(2 n V V^{\prime \prime}+(1-2 n) V^{\prime 2}\right)-4 n^{2}\left(t \frac{d \omega(t)}{d t}+\omega(t)+\kappa^{2} \alpha(t)\right) V^{2}+ \\
& 4 n^{2} \beta(t) V^{3}=0 .
\end{aligned}
$$

For the solutions of Eq. (27), let

$$
V(\xi)=\sum_{l=0}^{N} a_{l}\left(\frac{\psi^{\prime}(\xi)}{\psi(\xi)}\right)^{l}, a_{N} \neq 0,
$$

where $a_{l}$ are all constants to be determined. Balancing $V^{\prime \prime}$ with $V^{3}$ in Eq. (29) gives $N=2$. Consequently, we reach

$$
V(\xi)=a_{0}+a_{1}\left(\frac{\psi^{\prime}(\xi)}{\psi(\xi)}\right)+a_{2}\left(\frac{\psi^{\prime}(\xi)}{\psi(\xi)}\right)^{2} .
$$

Substituting Eq. (31) in Eq. (29) and then setting the coefficients of $\psi^{-j}, j=0,1,2,3,4,5,6$ to zero, then we obtain a set of algebraic equations involving $a_{0}, a_{1}, a_{2}, \kappa$ and $\omega(t)$ as following 
$\psi^{-6}$ Coeff.:

$$
4 a_{2}^{2}\left((n+1)(\alpha(t)+\gamma(t))+n^{2} \beta(t) a_{2}\right)\left(\psi^{\prime}\right)^{6}=0,
$$

$\psi^{-5}$ Coeff.:

$$
\begin{aligned}
& 4 a_{2}\left(\psi^{\prime}\right)^{4}\left(a_{1}\left((2 n+1)(\alpha(t)+\gamma(t))+3 n^{2} \beta(t) a_{2}\right) \psi^{\prime}-\right. \\
& \left.(n+2)(\alpha(t)+\gamma(t)) a_{2} \psi^{\prime \prime}\right)=0
\end{aligned}
$$

$\psi^{-4}$ Coeff.:

$$
\begin{aligned}
& \left(\psi^{\prime}\right)^{2}\left(a_{1}^{2}\left((2 n+1)(\alpha(t)+\gamma(t))+12 n^{2} \beta(t) a_{2}\right)\left(\psi^{\prime}\right)^{2}-\right. \\
& 2(4+5 n)(\alpha(t)+\gamma(t)) a_{1} a_{2} \psi^{\prime} \psi^{\prime \prime}+4 a_{2}\left(3 n a_{0}\left(\alpha(t)+\gamma(t)+n \beta(t) a_{2}\right)\left(\psi^{\prime}\right)^{2}+\right. \\
& a_{2}\left(-n^{2}\left(R+\alpha(t) \kappa^{2}\right)\left(\psi^{\prime}\right)^{2}-(n-1)(\alpha(t)+\gamma(t))\left(\psi^{\prime \prime}\right)^{2}+\right. \\
& \left.\left.\left.n(\alpha(t)+\gamma(t)) \psi^{\prime} \psi^{\prime \prime \prime}\right)\right)\right)=0,
\end{aligned}
$$

$\psi^{-3}$ Coeff.:

$$
\begin{aligned}
& 2 \psi^{\prime}\left(2 n a_{0} \psi^{\prime}\left(a_{1}\left(\alpha(t)+\gamma(t)+6 n \beta(t) a_{2}\right) \psi^{\prime}-5(\alpha(t)+\gamma(t)) a_{2} \psi^{\prime \prime}\right)+\right. \\
& a_{1}\left(2 n^{2} \beta a_{1}^{2}\left(\psi^{\prime}\right)^{2}-(n+1)(\alpha(t)+\gamma(t)) a_{1} \psi^{\prime} \psi^{\prime \prime}+\right. \\
& a_{2}\left(-4 n^{2}\left(t \frac{d \omega(t)}{d t}+\omega(t)+\alpha(t) \kappa^{2}\right)\left(\psi^{\prime}\right)^{2}-\right. \\
& \left.\left.\left.2(n-1)(\alpha(t)+\gamma(t))\left(\psi^{\prime \prime}\right)^{2}+3 n(\alpha(t)+\gamma(t)) \psi^{\prime} \psi^{\prime \prime \prime}\right)\right)\right)=0
\end{aligned}
$$

$\psi^{-2}$ Coeff.:

$$
\begin{aligned}
& -6 n(\alpha(t)+\gamma(t)) a_{0} a_{1} \psi^{\prime} \psi^{\prime \prime}+ \\
& 4 n a_{0} a_{2}\left(n\left(-2\left(t \frac{d \omega(t)}{d t}+\omega(t)+\alpha(t) \kappa^{2}\right)+3 \beta(t) a_{0}\right)\left(\psi^{\prime}\right)^{2}+\right. \\
& \left.(\alpha(t)+\gamma(t))\left(\psi^{\prime \prime}\right)^{2}+(\alpha(t)+\gamma(t)) \psi^{\prime} \psi^{(3)}\right)+ \\
& a_{1}^{2}\left(-4 n^{2}\left(t \frac{d \omega(t)}{d t}+\omega(t)+\alpha(t) \kappa^{2}-3 \beta a_{0}\right)\left(\psi^{\prime}\right)^{2}-\right. \\
& \left.(2 n-1)(\alpha(t)+\gamma(t))\left(\psi^{\prime \prime}\right)^{2}+2 n(\alpha(t)+\gamma(t)) \psi^{\prime} \psi^{\prime \prime \prime}\right)=0,
\end{aligned}
$$

$\psi^{-1}$ Coeff.:

$$
2 n a_{0} a_{1}\left(-2 n\left(2\left(t \frac{d \omega(t)}{d t}+\omega(t)+\alpha(t) \kappa^{2}\right)-3 \beta(t) a_{0}\right) \psi^{\prime}+(\alpha(t)+\gamma(t)) \psi^{\prime \prime \prime}\right)=0,
$$

$\psi^{0}$ Coeff.:

$$
4 n^{2} a_{0}^{2}\left(-t \frac{d \omega(t)}{d t}-\omega(t)-\alpha(t) \kappa^{2}+\beta(t) a_{0}\right)=0
$$

Solving this system, we obtain

$$
\begin{aligned}
& a_{0}=0, a_{2}=-\frac{(n+1)(\alpha(t)+\gamma(t))}{n^{2} \beta(t)} \\
& \omega(t)=\frac{1}{t} \int_{0}^{t}\left\{-4 \alpha(t) \kappa^{2}+\frac{n^{2} \beta(t)^{2} a_{1}^{2}}{4(n+1)^{2}(\alpha(t)+\gamma(t))}\right\} d t
\end{aligned}
$$

where $a_{1}$ is a real arbitrary constants. Substituting (39) into Eqs. (33) and (37), we obtain

$$
\begin{aligned}
\psi^{\prime \prime} & =\frac{n^{2} \beta(t) a_{1}}{(n+1)(\alpha(t)+\gamma(t))} \psi^{\prime}, \\
\psi^{\prime \prime \prime} & =\frac{n^{4} \beta(t)^{2} a_{1}^{2}}{(n+1)^{2}(\alpha(t)+\gamma(t))^{2}} \psi^{\prime} .
\end{aligned}
$$


From Eqs. (40) and (41), we can deduce that

$$
\begin{gathered}
\psi^{\prime}=\frac{(n+1)(\alpha(t)+\gamma(t))}{n^{2} \beta(t) a_{1}} c_{1} e^{\frac{n^{2} \beta(t) a_{1}}{(n+1)(\alpha(t)+\gamma(t))} \xi}, \\
\psi=\frac{(n+1)^{2}(\alpha(t)+\gamma(t))^{2}}{n^{4} \beta^{2}(t) a_{1}^{2}} c_{1} e^{\frac{n^{2} \beta(t) a_{1}}{(n+1)(\alpha(t)+\gamma(t))} \xi}+c_{2} .
\end{gathered}
$$

where $c_{1}$ and $c_{2}$ are constants of integration. Substituting Eqs. (42) and (43) into Eq. (31), we obtain the following new exact solution to Eq. (27)

$$
\begin{aligned}
& \psi(x, t)=\left\{\begin{array}{l}
a_{1}\left(\frac{\frac{(n+1)(\alpha(t)) \gamma(t))}{n^{2} \beta(t) a_{1}} c_{1} e^{\frac{n^{2} \beta(t) a_{1}}{(n+1)(\alpha(t)+\gamma(t))^{\xi}}}}{\frac{(n+1)^{2}(\alpha(t)+\gamma(t))^{2}}{n^{4} \beta^{2}(t) a_{1}^{2}} c_{1} e^{\frac{n^{2} \beta(t) a_{1}}{(n+1)(\alpha(t)+\gamma(t))^{\xi}}+c_{2}}}\right)- \\
\frac{(n+1)(\alpha(t)+\gamma(t))}{n^{2} \beta(t)}\left(\frac{\frac{(n+1)(\alpha(t)+\gamma(t))}{n^{2} \beta(t) a_{1}} c_{1} e^{\frac{n^{2} \beta(t) a_{1}}{(n+1)(\alpha(t)+\gamma(t))^{2}}}}{\frac{(n+1)^{2}(\alpha(t)+\gamma(t))^{2}}{n^{4} \beta^{2}(t) a_{1}^{2}} c_{1} e^{\frac{n^{2} \beta(t) a_{1}}{(n+1)(\alpha(t)+\gamma(t))^{\xi}}+c_{2}}}\right)^{2}
\end{array}\right\} \\
& \times e^{i\left(-\kappa x-\int_{0}^{t}\left\{4 \alpha(t) \kappa^{2}-\frac{n^{2} \beta(t)^{2} a_{1}^{2}}{4(n+1)^{2}(\alpha(t)+\gamma(t))}\right\} d t\right)} .
\end{aligned}
$$

If we set $c_{1}=\frac{n^{4} \beta(t)^{2} a_{1}^{2}}{(n+1)^{2}(\alpha(t)+\gamma(t))^{2}} e^{\frac{n^{2} \beta(t) a_{1}}{(n+1)(\alpha(t)+\gamma(t))} \xi_{0}}, c_{2}= \pm 1$, we obtain

$$
\begin{aligned}
& \psi(x, t)=\left\{\frac{n^{2} \beta(t) a_{1}^{2}}{4(n+1)(\alpha(t)+\gamma(t))} \operatorname{sech}^{2}\left[\frac{n^{2} \beta(t) a_{1}}{2(n+1)(\alpha(t)+\gamma(t))}\left(x+2 \kappa \int_{0}^{t} \alpha(t) d t+\xi_{0}\right)\right]\right\}^{\frac{1}{2 n}} \\
& \times e^{i\left(-\kappa x-\int_{0}^{t}\left\{4 \alpha(t) \kappa^{2}-\frac{n^{2} \beta(t) a_{1}^{2}}{4(n+1)^{2}(\alpha(t)+\gamma(t))}\right\} d t\right)} \\
& \psi(x, t)=\left\{-\frac{n^{2} \beta(t) a_{1}^{2}}{4(n+1)(\alpha(t)+\gamma(t))} \operatorname{csch}^{2}\left[\frac{n^{2} \beta(t) a_{1}}{2(n+1)(\alpha(t)+\gamma(t))}\left(x+2 \kappa \int_{0}^{t} \alpha(t) d t+\xi_{0}\right)\right]\right\}^{\frac{1}{2 n}} \\
& \times e^{i\left(-\kappa x-\int_{0}^{t}\left\{4 \alpha(t) \kappa^{2}-\frac{n^{2} \beta(t) a_{1}^{2}}{4(n+1)^{2}(\alpha(t)+\gamma(t))}\right\} d t\right)},
\end{aligned}
$$

\subsection{PARABOLIC LAW NONLINEARITY}

For parabolic-law nonlinearity, $G(s)=\beta s+\gamma s^{2}$, where $\beta$ and $\gamma$ are in general constants. Such as kind of nonlinearity appears also in nonlinear fiber optics. In this case, the RNLSE is [1-9]

$$
i \psi_{t}+\alpha(t) \psi_{x x}+\left\{\beta(t)|\psi|^{2}+\gamma(t)|\psi|^{4}\right\} \psi+d(t)\left(\frac{|\psi|_{x x}}{|\psi|}\right) \psi=0
$$

In this case, Eq. (47) reduces to

$$
(\alpha(t)+d(t)) U^{\prime \prime}-\left\{t \frac{d \omega(t)}{d t}+\omega(t)+\kappa^{2} \alpha(t)\right\} U+\beta(t) U^{3}+\gamma(t) U^{5}=0 .
$$

To obtain an analytic solution, we use the transformation $U=V^{\frac{1}{2}}$ in Eq. (48) to find

$$
\begin{aligned}
& (\alpha(t)+d(t))\left(2 V V^{\prime \prime}-\left(V^{\prime}\right)^{2}\right)-4\left(t \frac{d \omega(t)}{d t}+\omega(t)+\kappa^{2} \alpha(t)\right) V^{2}+ \\
& 4 \beta(t) V^{3}+4 \gamma(t) V^{4}=0 .
\end{aligned}
$$

For the solutions of Eq. (47), assume

$$
V(\xi)=\sum_{l=0}^{N} a_{l}\left(\frac{\psi^{\prime}(\xi)}{\psi(\xi)}\right)^{l}, a_{N} \neq 0,
$$


where $a_{l}$ are all constants to be determined. Balancing $V^{\prime \prime}$ with $V^{3}$ in Eq. (49) gives $N=1$. Consequently, we reach

$$
V(\xi)=a_{0}+a_{1}\left(\frac{\psi^{\prime}(\xi)}{\psi(\xi)}\right)
$$

Substituting Eq. (51) in Eq. (49) and then setting the coefficients of $\psi^{-j}, j=0,1,2,3,4$ to zero, then we obtain a set of algebraic equations involving $a_{0}, a_{1}, a_{2}, \kappa$ and $\omega(t)$ as following $\psi^{-4}$ Coeff.:

$$
a_{1}^{2}\left(3(\alpha(t)+d(t))+4 \gamma(t) a_{1}^{2}\right)\left(\psi^{\prime}\right)^{4}=0,
$$

$\psi^{-3}$ Coeff:

$$
4 a_{1}\left(\psi^{\prime}\right)^{2}\left(a_{0}\left(\alpha(t)+d(t)+4 \gamma(t) a_{1}^{2}\right) \psi^{\prime}+a_{1}\left(\beta(t) a_{1} \psi^{\prime}-(\alpha(t)+d(t)) \psi^{\prime \prime}\right)\right)=0,
$$

$\psi^{-2}$ Coeff.:

$$
\begin{aligned}
& -a_{1}\left(6(\alpha(t)+d(t)) a_{0} \psi^{\prime} \psi^{\prime \prime}+a_{1}\left(4\left(t \frac{d \omega(t)}{d t}+\omega(t)+\alpha(t) \kappa^{2}-3 \beta(t) a_{0}-6 \gamma(t) a_{0}^{2}\right)\left(\psi^{\prime}\right)^{2}+\right.\right. \\
& \left.\left.(\alpha(t)+d(t))\left(\psi^{\prime \prime}\right)^{2}-2(\alpha(t)+d(t)) \psi^{\prime} \psi^{\prime \prime \prime}\right)\right)=0,
\end{aligned}
$$

$\psi^{-1}$ Coeff.:

$$
2 a_{0} a_{1}\left(\left(-4\left(t \frac{d \omega(t)}{d t}+\omega(t)+\alpha(t) \kappa^{2}\right)+6 \beta(t) a_{0}+8 \gamma(t) a_{0}^{2}\right) \psi^{\prime}+(\alpha(t)+d(t)) \psi^{\prime \prime \prime}\right)=0,
$$

$\psi^{0}$ Coeff.:

$$
4 a_{0}^{2}\left(-t \frac{d \omega(t)}{d t}-\omega(t)-\alpha(t) \kappa^{2}+\beta(t) a_{0}+\gamma(t) a_{0}^{2}\right)=0
$$

Solving this system, we obtain

$$
a_{0}=\frac{-3 \beta(t)}{4 \gamma(t)}, \quad a_{1}= \pm \sqrt{\frac{-3(\alpha(t)+d(t))}{4 \gamma(t)}}, \omega(t)=\frac{1}{t} \int_{0}^{t}\left\{-\frac{3 \beta^{2}(t)}{16 \gamma(t)}-\alpha(t) \kappa^{2}\right\} d t
$$

where $a_{1}$ is a real arbitrary constants. Substituting (51) into Eqs. (53) and (55), we obtain

$$
\begin{gathered}
\psi^{\prime \prime}= \pm \sqrt{\frac{-3 \beta^{2}(t)}{4(\alpha(t)+d(t)) \gamma(t)}} \psi^{\prime}, \\
\psi^{\prime \prime \prime}=\frac{-3 \beta^{2}(t)}{4(\alpha(t)+d(t)) \gamma(t)} \psi^{\prime} .
\end{gathered}
$$

From Eqs. (58) and (59), we can deduce that

$$
\begin{aligned}
& \psi^{\prime}= \pm \sqrt{\frac{-4(\alpha(t)+d(t)) \gamma(t)}{3 \beta^{2}(t)}} c_{1} e^{ \pm \sqrt{\frac{-3 \beta^{2}(t)}{4(\alpha(t)+d(t)) \gamma(t)}}}, \\
& \psi=\frac{-4(\alpha(t)+d(t)) \gamma(t)}{3 \beta^{2}(t)} c_{1} e^{ \pm \sqrt{\frac{-3 \beta^{2}(t)}{4(\alpha(t)+d(t)) \gamma(t)}}} \xi c_{2} .
\end{aligned}
$$


where $c_{1}$ and $c_{2}$ are constants of integration. Substituting Eqs. (60) and (61) into Eq. (51), we obtain the following new exact solution to Eq. (47)

$$
\begin{aligned}
& \psi(x, t)=\left(\frac{-3 \beta(t)}{4 \gamma(t)} \pm \sqrt{\frac{-3(\alpha(t)+d(t))}{4 \gamma(t)}}\left\{\frac{ \pm \sqrt{\frac{-4(\alpha(t)+d(t)) \gamma(t)}{3 \beta^{2}(t)}} c_{1} e^{ \pm \sqrt{\frac{-3 \beta^{2}(t)}{4(\alpha(t)+d(t)) \gamma(t)}}}}{\frac{-4(\alpha(t)+d(t)) \gamma(t)}{3 \beta^{2}(t)} c_{1} e^{ \pm \sqrt{\frac{-3 \beta^{2}(t)}{4(\alpha(t)+d(t) \gamma(t)}} \xi}+c_{2}}\right\}\right) \\
& \times e^{i\left(-\kappa x-\int_{0}^{t}\left\{\frac{3 \beta^{2}(t)}{16 \gamma(t)}+\alpha(t) \kappa^{2}\right\} d t\right)} .
\end{aligned}
$$

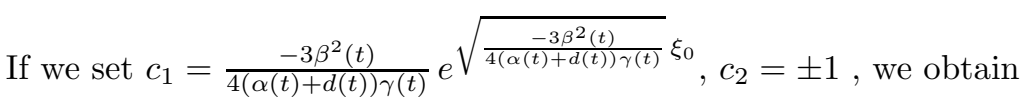

$$
\begin{aligned}
& \psi(x, t)=\left(\frac{-3 \beta(t)}{8 \gamma(t)}\left\{1 \mp \tanh \left[\frac{1}{2} \sqrt{\frac{-3 \beta^{2}(t)}{4 \gamma(t)(\alpha(t)+d(t))}}\left(x+2 \kappa \int_{0}^{t} \alpha(t) d t+\xi_{0}\right)\right]\right\}\right)^{\frac{1}{2}} \\
& \times e^{i\left(-\kappa x-\int_{0}^{t}\left\{\frac{\beta \beta^{2}(t)}{16 \gamma(t)}+\alpha(t) \kappa^{2}\right\} d t\right)}, \\
& \psi(x, t)=\left(\frac{-3 \beta(t)}{8 \gamma(t)}\left\{1 \mp \operatorname{coth}\left[\frac{1}{2} \sqrt{\frac{-3 \beta^{2}(t)}{4 \gamma(t)(\alpha(t)+d(t))}}\left(x+2 \kappa \int_{0}^{t} \alpha(t) d t+\xi_{0}\right)\right]\right\}\right)^{\frac{1}{2}} \\
& \times e^{i\left(-\kappa x-\int_{0}^{t}\left\{\frac{3 \beta^{2}(t)}{16 \gamma(t)}+\alpha(t) \kappa^{2}\right\} d t\right)},
\end{aligned}
$$

\subsection{DUAL-POWER LAW NONLINEARITY}

For dual-power law nonlinearity, $G(s)=\beta s^{n}+\gamma s^{2 n}$, where $\beta$ and $\gamma$ are in general constants although in this paper they are taken to be time-dependent. This nonlinearity serves as a basic model to describe the solitons in photovoltaic-photorefractive materials such as $\mathrm{LiNbO}_{3}$. Moreover, this law is a generalization of the parabolic law nonlinearity. In fact, setting $n=1$, the dual-power law nonlinearity. In this case, the RNLSE is [1-9]

$$
i \psi_{t}+\alpha(t) \psi_{x x}+\left\{\beta(t)|\psi|^{2 n}+\gamma(t)|\psi|^{4 n}\right\} \psi+d(t)\left(\frac{|\psi|_{x x}}{|\psi|}\right) \psi=0 .
$$

In this case, Eq. (65) reduces to

$$
(\alpha(t)+d(t)) U^{\prime \prime}-\left\{t \frac{d \omega(t)}{d t}+\omega(t)+\kappa^{2} \alpha(t)\right\} U+\beta(t) U^{2 n+1}+\gamma(t) U^{4 n+1}=0 .
$$

To obtain an analytic solution, we use the transformation $U=V^{\frac{1}{2 n}}$ in Eq. (66) to find

$$
\begin{aligned}
& (\alpha(t)+d(t))\left(2 n V V^{\prime \prime}+(1-2 n)\left(V^{\prime}\right)^{2}\right)-4 n^{2}\left(t \frac{d \omega(t)}{d t}+\omega(t)+\kappa^{2} \alpha(t)\right) V^{2}+ \\
& 4 n^{2} \beta(t) V^{3}+4 n^{2} \gamma(t) V^{4}=0 .
\end{aligned}
$$

For the solutions of Eq. (65), we assume:

$$
V(\xi)=\sum_{l=0}^{N} a_{l}\left(\frac{\psi^{\prime}(\xi)}{\psi(\xi)}\right)^{l}, a_{N} \neq 0,
$$

where $a_{l}$ are all constants to be determined. Balancing $V^{\prime \prime}$ with $V^{3}$ in Eq. (67) gives $N=1$. Consequently, we reach

$$
V(\xi)=a_{0}+a_{1}\left(\frac{\psi^{\prime}(\xi)}{\psi(\xi)}\right)
$$


Substituting Eq. (69) in Eq. (67) and then setting the coefficients of $\psi^{-j}, j=0,1,2,3,4$ to zero, then we obtain a set of algebraic equations involving $a_{0}, a_{1}, a_{2}, \kappa$ and $\omega(t)$ as following $\psi^{-4}$ Coeff.:

$$
a_{1}^{2}\left((1+2 n)(\alpha(t)+d(t))+4 n^{2} \gamma(t) a_{1}^{2}\right)\left(\psi^{\prime}\right)^{4}=0
$$

$\psi^{-3}$ Coeff.:

$$
\begin{aligned}
& 2 a_{1}\left(\psi^{\prime}\right)^{2}\left(2 n a_{0}\left(\alpha(t)+d(t)+4 n \gamma(t) a_{1}^{2}\right) \psi^{\prime}+\right. \\
& \left.a_{1}\left(2 n^{2} \beta(t) a_{1} \psi^{\prime}-(1+n)(\alpha(t)+d(t)) \psi^{\prime \prime}\right)\right)=0,
\end{aligned}
$$

$\psi^{-2}$ Coeff:

$$
\begin{aligned}
& a_{1}\left(-6 n(\alpha(t)+d(t)) a_{0} \psi^{\prime} \psi^{\prime \prime}+\right. \\
& a_{1}\left(-4 n^{2}\left(t \frac{d \omega(t)}{d t}+\omega(t)+\alpha(t) \kappa^{2}-3 \beta(t) a_{0}-6 \gamma(t) a_{0}^{2}\right)\left(\psi^{\prime}\right)^{2}-\right. \\
& \left.\left.(-1+2 n)(\alpha(t)+d(t))\left(\psi^{\prime \prime}\right)^{2}+2 n(\alpha(t)+d(t)) \psi^{\prime} \psi^{\prime \prime \prime}\right)\right)=0
\end{aligned}
$$

$\psi^{-1}$ Coeff.:

$$
2 n a_{0} a_{1}\left(2 n\left(-2\left(t \frac{d \omega(t)}{d t}+\omega(t)+\alpha(t) \kappa^{2}\right)+3 \beta(t) a_{0}+4 \gamma(t) a_{0}^{2}\right) \psi^{\prime}+(\alpha(t)+d(t)) \psi^{\prime \prime \prime}\right)=0,
$$

$\psi^{-2}$ Coeff.:

$$
4 n^{2} a_{0}^{2}\left(-t \frac{d \omega(t)}{d t}-\omega(t)-\alpha(t) \kappa^{2}+\beta(t) a_{0}+\gamma(t) a_{0}^{2}\right)=0
$$

Solving this system, we obtain

$$
\begin{aligned}
& a_{0}=\frac{-(2 n+1) \beta(t)}{2(n+1) \gamma(t)}, \quad a_{1}= \pm \sqrt{\frac{-(2 n+1)(\alpha(t)+d(t))}{4 n^{2} \gamma(t)}}, \\
& \omega(t)=\frac{1}{t} \int_{0}^{t}\left\{-\frac{(2 n+1) \beta^{2}(t)}{4(n+1)^{2} \gamma(t)}-\alpha(t) \kappa^{2}\right\} d t
\end{aligned}
$$

where $a_{1}$ is a real arbitrary constants. Substituting (69) into Eqs. (73) and (71), we obtain

$$
\begin{gathered}
\psi^{\prime \prime}= \pm \sqrt{\frac{-n^{2}(2 n+1) \beta^{2}(t)}{(n+1)^{2}(\alpha(t)+d(t)) \gamma(t)}} \psi^{\prime}, \\
\psi^{\prime \prime \prime}=\frac{-n^{2}(2 n+1) \beta^{2}(t)}{(n+1)^{2}(\alpha(t)+d(t)) \gamma(t)} \psi^{\prime} .
\end{gathered}
$$

From Eqs. (75) and (76), we can deduce that

$$
\begin{aligned}
& \psi^{\prime}= \pm \sqrt{\frac{-(n+1)^{2}(\alpha(t)+d(t)) \gamma(t)}{n^{2}(2 n+1) \beta^{2}(t)}} c_{1} e^{ \pm \sqrt{\frac{-n^{2}(2 n+1) \beta^{2}(t)}{(n+1)^{2}(\alpha(t)+d(t)) \gamma(t)}}} \xi \\
& \psi=\frac{-(n+1)^{2}(\alpha(t)+d(t)) \gamma(t)}{n^{2}(2 n+1) \beta^{2}(t)} c_{1} e^{ \pm \sqrt{\frac{-n^{2}(2 n+1) \beta^{2}(t)}{(n+1)^{2}(\alpha(t)+d(t)) \gamma(t)}} \xi}+c_{2} .
\end{aligned}
$$

where $c_{1}$ and $c_{2}$ are constants of integration. Substituting Eqs. (77) and (78) into Eq. (69), we obtain the following new exact solution to Eq.(65)

$$
\begin{aligned}
& \psi(x, t)=\left(\frac{-(2 n+1) \beta(t)}{2(n+1) \gamma(t)} \pm \sqrt{\frac{-(2 n+1)(\alpha(t)+d(t))}{4 n^{2} \gamma(t)}}\left\{\frac{ \pm \sqrt{\frac{-(n+1)^{2}(\alpha(t)+d(t)) \gamma(t)}{n^{2}(2 n+1) \beta^{2}(t)}} c_{1} e^{ \pm \sqrt{\frac{-n^{2}(2 n+1) \beta^{2}(t)}{(n+1)^{2}(\alpha(t)+d(t) \gamma(t)}} \xi}}{\frac{-(n+1)^{2}(\alpha(t)+d(t)) \gamma(t)}{n^{2}(2 n+1) \beta^{2}(t)} c_{1} e^{ \pm \sqrt{\frac{-n^{2}(2 n+1) \beta^{2}(t)}{(n+1)^{2}(\alpha(t)+d(t)) \gamma(t)}} \xi}+c_{2}}\right\}\right) \\
& \times e^{i\left(-\kappa x-\int_{0}^{t}\left\{\frac{(2 n+1) \beta^{2}(t)}{4(n+1)^{2} \gamma(t)}+\alpha(t) \kappa^{2}\right\} d t\right)} .
\end{aligned}
$$


If we set $c_{1}=\frac{-n^{2}(2 n+1) \beta^{2}(t)}{(n+1)^{2}(\alpha(t)+d(t)) \gamma(t)} e^{\sqrt{\frac{-n^{2}(2 n+1) \beta^{2}(t)}{(n+1)^{2}(\alpha(t)+d(t)) \gamma(t)}} \xi_{0}}, c_{2}= \pm 1$, we obtain

$$
\begin{aligned}
& \psi(x, t)=\left(\frac{-(2 n+1) \beta(t)}{4(n+1) \gamma(t)}\left\{1 \mp \tanh \left[\frac{1}{2} \sqrt{\frac{-n^{2}(2 n+1) \beta^{2}(t)}{(n+1)^{2}(\alpha(t)+d(t)) \gamma(t)}}\left(x+2 \kappa \int_{0}^{t} \alpha(t) d t+\xi_{0}\right)\right]\right\}\right)^{\frac{1}{2 n}} \\
& \times e^{i\left(-\kappa x-\int_{0}^{t}\left\{\frac{(2 n+1) \beta^{2}(t)}{4(n+1)^{2} \gamma(t)}+\alpha(t) \kappa^{2}\right\} d t\right)} \\
& \psi(x, t)=\left(\frac{-(2 n+1) \beta(t)}{4(n+1) \gamma(t)}\left\{1 \mp \operatorname{coth}\left[\frac{1}{2} \sqrt{\frac{-n^{2}(2 n+1) \beta^{2}(t)}{(n+1)^{2}(\alpha(t)+d(t)) \gamma(t)}}\left(x+2 \kappa \int_{0}^{t} \alpha(t) d t+\xi_{0}\right)\right]\right\}\right)^{\frac{1}{2 n}} \\
& \times e^{i\left(-\kappa x-\int_{0}^{t}\left\{\frac{(2 n+1) \beta^{2}(t)}{4(n+1)^{2} \gamma(t)}+\alpha(t) \kappa^{2}\right\} d t\right)},
\end{aligned}
$$

\section{CONCLUSIONS}

This paper revisited RNLSE with time-dependent coefficients that comes with four types of nonlinearity. The study was devoted to the application of modified simple equation method. This resulted in bright, dark and singular soliton solutions. The solitons exist with several constraint conditions that naturally appear from the solution structure. Another important aspect for these solitons to exist is that the time-dependent coefficients must be Riemann integrable. The results of this paper are indeed encouraging. Later, these results will be explored further. Besides time-dependent coefficients, stochastic coefficients will be considered in future. This will lead to additional interesting results. Furthermore, fractional temporal evolution will be studied. The results of those research will be reported as well.

\section{ACKNOWLEDGMENT}

The third author (QZ) was funded by the National Science Foundation of Hubei Province in China under the grant number 2015CFC891. The fourth author (SPM) would like to thank the research support provided by the Department of Mathematics and Statistics at Tshwane University of Technology and the support from the South African National Foundation under Grant Number 92052 IRF1202210126. The fifth author (AB) would like to thank Tshwane University of Technology during his academic visit during 2016. The research work of fifth and sixth authors (AB \& MB) were supported by Qatar National Research Fund (QNRF) under the grant number NPRP 6-021-1-005. The authors also declare that there is no conflict of interest. 


\section{References}

[1] A. H. Arnous, M. Mirzazadeh, Q. Zhou, M. F. Mahmood, A. Biswas \& M. Belic. "Optical solitons with resonant nonlinear Schrödinger's equation using $G^{\prime} / G$-expansion scheme". Optoelectronics and Advanced Materials - Rapid Communications. Volume 9, Numbers 9-10, 1214-1220. (2015).

[2] A. Biswas. "Soliton solution of the perturbed resonant nonlinear Schrödinger's equation with full nonlinearity by semi-inverse variational principle". Quantum Physics Letters. Volume 1, Number 2, 79-84. (2012).

[3] M. Eslami, M. Mirzazadeh \& A. Biswas. "Soliton solutions of the resonant nonlinear Schrödinger's equation in optical fibers with time-dependent coefficients by simplest equation approach". Journal of Modern Optics. Volume 60, Issue 19, 1627-1636. (2013).

[4] M. Eslami, M. Mirzazadeh, F. Vajargah \& A. Biswas. "Optical solitons for resonant nonlinear Schrödinger's equation with time-dependent coefficients by the first integral method". Optik. Volume 125, Issue 13, 3107-3116. (2014).

[5] M. Eslami, M. Mirzazadeh, F. Vajargah \& A. Biswas. "Optical solitons and optical rogons of generalized resonant dispersive nonlinear Schrödinger's equation with power law nonlinearity". Optik. Volume 125, Issue 16, 4246-4256. (2014).

[6] M. Mirzazadeh, M. Eslami, D. Milovic \& A. Biswas. "Topological solitons of resonant nonlinear Schrödinger's equation with dual-power law nonlinearity using $G^{\prime} / G$-expansion technique". Optik. Volume 125, Issue 19, 5480-5489. (2014).

[7] M. Mirzazadeh, A. H. Arnous, M. F. Mahmood, E. Zerrad \& A. Biswas. "Soliton solutions to resonant nonlinear Schrödinger's equation with time-dependent coefficients by trial solutions approach". Nonlinear Dynamics. Volume 81, Issue 1-2, 277-282. (2015).

[8] M. Mirzazadeh, Q. Zhou, A. Biswas \& M. Belic "Optical solitons of generalized dispersive nonlinear Schrödinger's equation with power law nonlinearity". Optoelectronics and Advanced Materials - Rapid Communications. Volume 9, Numbers 11-12, 1347-1352. (2015).

[9] M. Mirzazadeh, Q. Zhou, E. Zerrad, M. F. Mahmood, A. Biswas \& M. Belic "Bifurcation analysis and bright soliton of generalized resonant dispersive nonlinear Schrödinger's equation". Optoelectronics and Advanced Materials - Rapid Communications. Volume 9, Numbers 9-10, 1214-1220. (2015).

[10] A. J. M. Jawad, M. D. Petkovic \& A. Biswas. "Modified simple equation method for nonlinear evolution equations". Applied Mathematics and Computation. Volume 217, 869-877. (2010).

[11] H. Triki, T. Hayat, O. M. Aldossary \& A. Biswas. "Bright and dark solitons for the resonant nonlinear Schrödinger's equation with time-dependent coefficients". Optics and Laser Technology. Volume 44, Issue 7, 2223-2231. (2012).

[12] H. Triki, A. Yildirim, T. Hayat, O. M. Aldossary \& A. Biswas. "1-soliton solution of the generalized dispersive nonlinear Schrödinger's equation with time-dependent coefficients". Advanced Science Letters. Volume 16, 309-312. (2012).

[13] H. Triki \& A. M. Wazwaz. "New solitons nd periodic solutions for the (2+1)-dimensional Heisenburg ferromagnetic spin chain equation". To appear in Journal of Electromagnetic Waves and Applications. 
[14] H. Triki \& A. M. Wazwaz. "Trial equation method for solving the generalized Fisher's equation with variable coefficients". To appear in Physics Letters A.

[15] H. Triki \& A. M. Wazwaz. "Soliton solutions of the cubic-quintic nonlinear Schrödinger's equation with variable coefficients". Romanian Journal of Physics. Volume 61, Issies 3-4, 360-366. (2016).

[16] A. M. Wazwaz. "A seventh-order member of KdV6 hierarchy and its (2+1)-dimensional extensions". To appear in Modern Physics Letters B.

[17] A. M. Wazwaz. "Gaussian solitary waves for logarithmic-BBM and logarithmic-TRLW equations". To appear in Journal of Mathematical Chemistry.

[18] A. M. Wazwaz. "Kadomtsev-Petviashvili hierarchy: $N$-soliton solutions and ditinct dispersion relations". Applied Mathematics Letters. Volume 45, 86-92. (2015).

[19] A. M. Wazwaz. "Gaussian solitary waves for nonlinear evolution equations with logarithmic nonlinearities". Nonlinear Dynamics. Volume 83, 591-596. (2016).

[20] E. M. E. Zayed \& A. H. Arnous. "Exact solutions of the nonlinear ZK-MEW and the potential YTSF equations using the modified simple equation method". AIP Conference Proceedings. Volume 1479, 2044-2048. (2012). 